\title{
Calcifying fibrous pseudotumor in the pelvic cavity: A case report and review of the literature
}

\author{
HONG-YU MA ${ }^{1}$, MING-TAO FENG ${ }^{2}$ and YONG-GANG HONG ${ }^{1}$ \\ Departments of ${ }^{1}$ Colorectal Surgery and ${ }^{2}$ Neurosurgery, Changhai Hospital, Shanghai 200433, P.R. China
}

Received June 4, 2019; Accepted October 25, 2019

DOI: $10.3892 / \mathrm{mco} .2020 .1976$

\begin{abstract}
Calcifying fibrous pseudotumors (CFPs) are rare benign lesions mostly occurring in the limbs, necks and gastrointestinal tracts of young adults. In the present study, we report the first known case of a pelvic CFP in China in a 67-year-old male patient. The solitary tumor had an extremely regular shape and was in the vicinity of the sigmoid colon. Surgical resection was performed and the tumor was histologically diagnosed as CFP. We reviewed 64 cases of CFPs and discussed the clinical features of this disease in China and other countries. We found that the CFPs were most common in patients aged 18-60 and often had no obvious symptoms. They were immunoreactive for vimentin and factor XIIIa, while negative for CD117 and S100. In China, CFPs were mostly identified in the stomach, possibly associated with Chinese eating. The pathogenesis of this disease remains unclear. More research is needed to confirm the present results.
\end{abstract}

\section{Introduction}

Calcifying fibrous pseudotumor (CFP) are rare benign lesions first reported by Rosenthal and Abdul-Karim in 1988 (1). Pelvic CFP was first reported by Saglam et al in 2005 (2). Recently, we found an interesting CFP in the pelvic cavity. It was the first case of pelvic CFP in China. Because of the low morbidity and non-significant clinical symptoms, preoperative diagnosis of CFP is difficult. Here we provide the clinical and pathological features of this tumor, and we review CFP cases reported over the past ten years. We also compared the characteristics of this

Correspondence to: Dr Yong-Gang Hong, Department of Colorectal Surgery, Changhai Hospital, 168 Changhai Road, Shanghai 200433, P.R. China

E-mail: hyg810@163.com

Abbreviations: CFP, calcifying fibrous pseudotumor; SMA, smooth muscle actin; GIST, gastrointestinal stromal tumor; IMT, inflammatory myofibroblastic tumor; SFT, solitary fibrous tumors; $\mathrm{N}$, not applicable; +, positive; -, negative; \pm positive or negative

Key words: calcifying fibrous pseudotumor, immunohistochemistry, gastrointestinal tract disease between China and other countries, hoping to provide new concepts for clinical diagnosis and treatment.

\section{Case report}

A 67-year-old man presented with mild abdominal discomfort on 1 month duration. The patient had no history of trauma or surgery. Physical examination suggested a $1.5 \times 2.0 \mathrm{~cm}$ lump in the left lower abdomen, characterized as hard, painless, and movable. An enhanced abdominal CT revealed a $3.76 \times 3.44 \mathrm{~cm}$ mass near the sigmoid colon, with a smooth edge and a hyper-dense center (Fig. 1). No obvious enhancement appeared after contrast injection. Exploratory laparotomy and lumpectomy was performed under general anesthesia. Intraoperative exploration revealed a solitary, well-circumscribed, tough mass near the sigmoid colon. Lymph nodes in the mesentery were normal. The shape of the tumor was unusual (Fig. 2A and B). There was an extremely smooth and regular surface. The cross-section of the tumor was uniformly greyish white. A large calcification lesion with a diameter of $1 \mathrm{~cm}$ was located in the central part of the tumor, surrounded by abundant fibrous tissues. Histological examination revealed that the tumor was mainly composed by hyaline degeneration tissues and multifocal calcification. Few cells were detected except for some infiltrating lymphocytes and foamy cells (Fig. 2C and D). Immunohistochemistry showed vimentin(+), smooth muscle actin(+), CD34(+), CD117(-), and S-100 protein(-). The mass was surgically removed without complication. At 1-year follow-up, the patient showed no recurrence and was physically intact.

This study was approved by the Institutional Review Board of Ethics Committee of Changhai Hospital (Shanghai, China). We retrospectively analyzed 64 patients with CFPs over the past 10 years. One patient was admitted to our hospital at October 3, 2018. The patient gave written informed consent to appear in study. The other 63 patients were obtained by searching PubMed. Fifteen were reported by Chinese authors and 48 were from other countries. The search terms 'calcifying fibrous pseudotumor' and 'calcifying fibrous tumor' were used in our literature search. Definite diagnoses of CFP in these patients were obtained. All eligible studies with full-text articles were included. Data were managed with microsoft excel.

A total of 64 patients were retrospectively analyzed. There were more male than female patients in China, but no significant differences overall. The mean age of the patients 

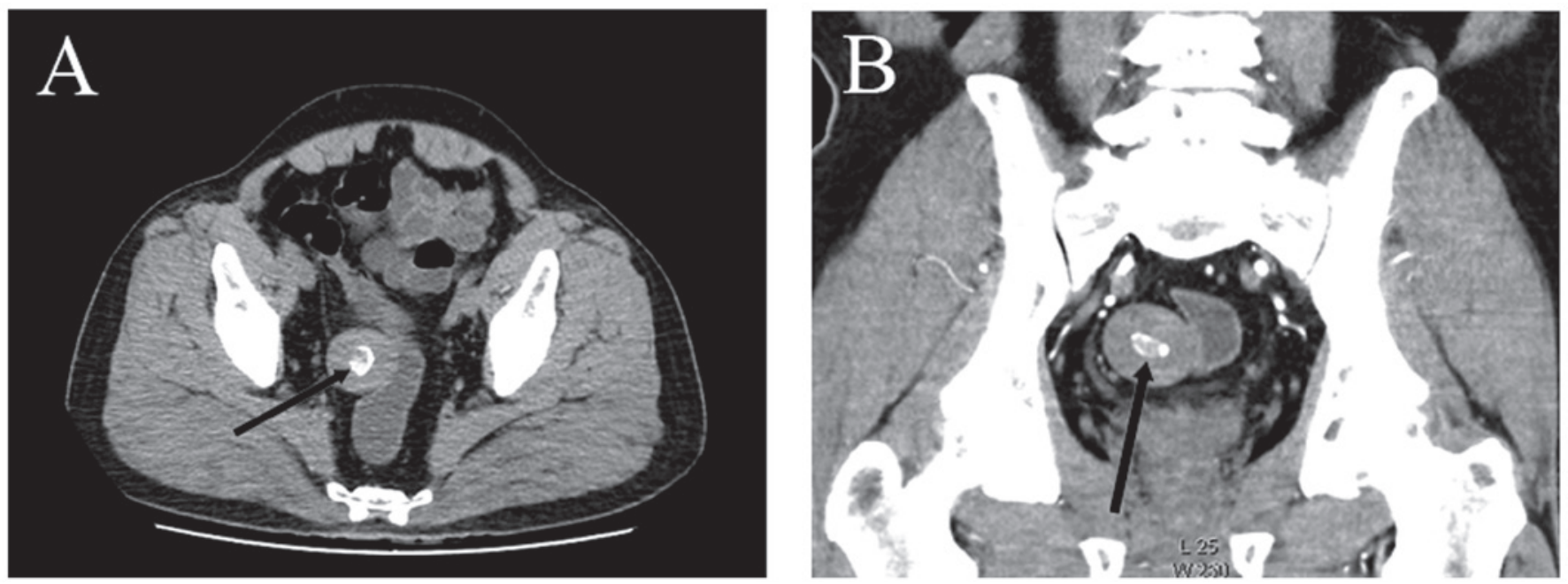

Figure 1. CT findings. (A) Axial view of the abdominal cavity CT. (B) Coronal view of the abdominal cavity CT. CT, computed tomography.

was 39.2 (range $8-77$ years). Over $80 \%$ of patients were from 18 to 60 years old. The disease has no characteristic symptoms; $48.4 \%$ patients discovered the tumors incidentally without obvious symptoms; $31.3 \%$ of patients presented with abdominal discomfort, and $20.3 \%$ of patients suffered from other symptoms (Table I). Abdominal discomfort was most common in Chinese patients, accounting for $43.6 \%$. The majority of patients had single lumps, and the mean diameter was $3.7+2.3$, with regular or irregular shape (Table II). The tumors were most seen in stomach (19 patients, 29.7\%), intestine (13 patients, 20.3\%) and thorax (12 patients, 18.7\%). Among the 16 patients from China, 7 (41.2\%) presented with stomach CFPs. Immunohistochemically, only some articles reported immunohistochemistry results and the measured parameters also varied. CFP cells were diffusely positive for vimentin and factor XIII (30 patients for vimentin and 15 for factor XIII. Tumors in 20 of $42(47.6 \%)$ patients stained focally with CD34. Tumors in 11 of $27(40.7 \%)$ cases were partially positive for smooth muscle actin (SMA). Tumors in 6 of 29 (20.7\%) patients were variably positive for desmin. Almost all tumors were negative for CD117 and S-100 protein. In terms of treatment, all patients included were treated with surgical removal. During follow-up, only one patient showed recurrence of the tumor.

\section{Discussion}

CFP is a rare benign tumor identified by the World Health Organization in 2002 (1). The tumor is common in children and adolescents (3), and is mostly found in skin, neck, pleura (4), abdominal cavity, as well as in internal organs such as the gastrointestinal tract (5), adrenal gland (6), and lung (7). Patients typically have no obvious symptoms. The tumors are often detected incidentally during imaging examinations or surgery.

The pathogenesis of CFP is not clear. Tumors in patients with a history of trauma and surgery are considered as a result of excessive postoperative inflammation responses (8). Larson et al suggested that CFP could be an unrecognized lesion of IgG4-related disease (9); however, Shimizu et al reported a patient with no plasma cells containing IgG4 in the tumor (10). Mehrad et al found copy number losses on chromosome 8 and deleterious mutations in ZN717, FRG1, and CDC27 genes, all of which are a novel findings for studies of CFTP tumorigenesis (11).

We analyzed 64 cases and found that the disease may be more common in young and middle aged (18-60-year-old) males. Symptoms are atypical and most likely induced by the tumor compressing surrounding tissues. CFPs in the abdominal cavity and gastrointestinal tract might lead to indigestion, bloating and abdominal pain, while those in thorax could induce pleural effusion. Abdominal discomfort was more common in China (43.6\%) than other countries $(27.1 \%)$, while $41.2 \%$ patients in China presented with stomach masses and in other countries, the proportion was $25 \%$. It has been suggested that abdominal discomfort is possibly related to stomach lesions. Dietary differences between China and other countries might explain the differences, and more studies are needed to confirm this. Among the included cases, the mean diameter of the tumor was $3.7 \mathrm{~cm}$. The shapes of the tumors were considered to be related to their position. We found that the CFPs from the gastrointestinal tract and abdominal cavity were solitary, regular, with typical appearance on CT. Most of the tumors in the thoracic cavity and arms were small, scattered, and irregular.

Imaging is important for primary diagnosis of CFP. In most cases, CT reveals that CFPs appear regular isodense masses with hyper-dense cores of calcification. Diffuse hyper-dense signals could also be detected in tumors with scattered calcification lesions (12). On MRI, the lesion had a hypointense signal on $\mathrm{T} 1$ and $\mathrm{T} 2$ weighted imaging, but an isodensity signal on gadolinium-enhanced T1 weighted imaging (13). In addition, tumors from gastrointestinal wall could be detected directly using barium gastroenterography and gastrointestinal endoscopy.

On gross inspection, CFPs are characterized as homogenous and hypovascular masses. These masses were composed of dense and hyalinized stroma, with scattered strips of calcification. Under microscopic observation, hypocellular sclerosis and coarse collagen were mostly 
Table I. Patient characteristics.

\begin{tabular}{lrrr}
\hline Variables & China $(\%)$ & Other countries $(\%)$ & Total $(\%)$ \\
\hline Sex & & & $24(50.0)$ \\
Male & $10(62.5)$ & $24(50.0)$ & $34(53.1)$ \\
Female & $6(37.5)$ & & $30(46.9)$ \\
Age & & $2(4.2)$ & $3(4.7)$ \\
$0-17$ & $1(6.3)$ & $43(89.5)$ & $56(87.5)$ \\
$18-60$ & $13(81.2)$ & $3(6.3)$ & $5(7.8)$ \\
$>60$ & $2(12.5)$ & $28(58.3)$ & $3(48.4)$ \\
Symptoms & & $13(27.1)$ & $20(31.3)$ \\
No obvious symptoms & $3(18.8)$ & $2(4.2)$ & $5(7.8)$ \\
Abdominal discomfort & $7(43.6)$ & $1(2.1)$ & $2(3.1)$ \\
Painless nodules & $3(18.8)$ & $3(6.2)$ & $2(6.3)$ \\
Shortness of breath & $1(6.3)$ & $1(2.1)$ & \\
Chest pain & $1(6.3)$ & & \\
Other symptoms & $1(6.3)$ & &
\end{tabular}
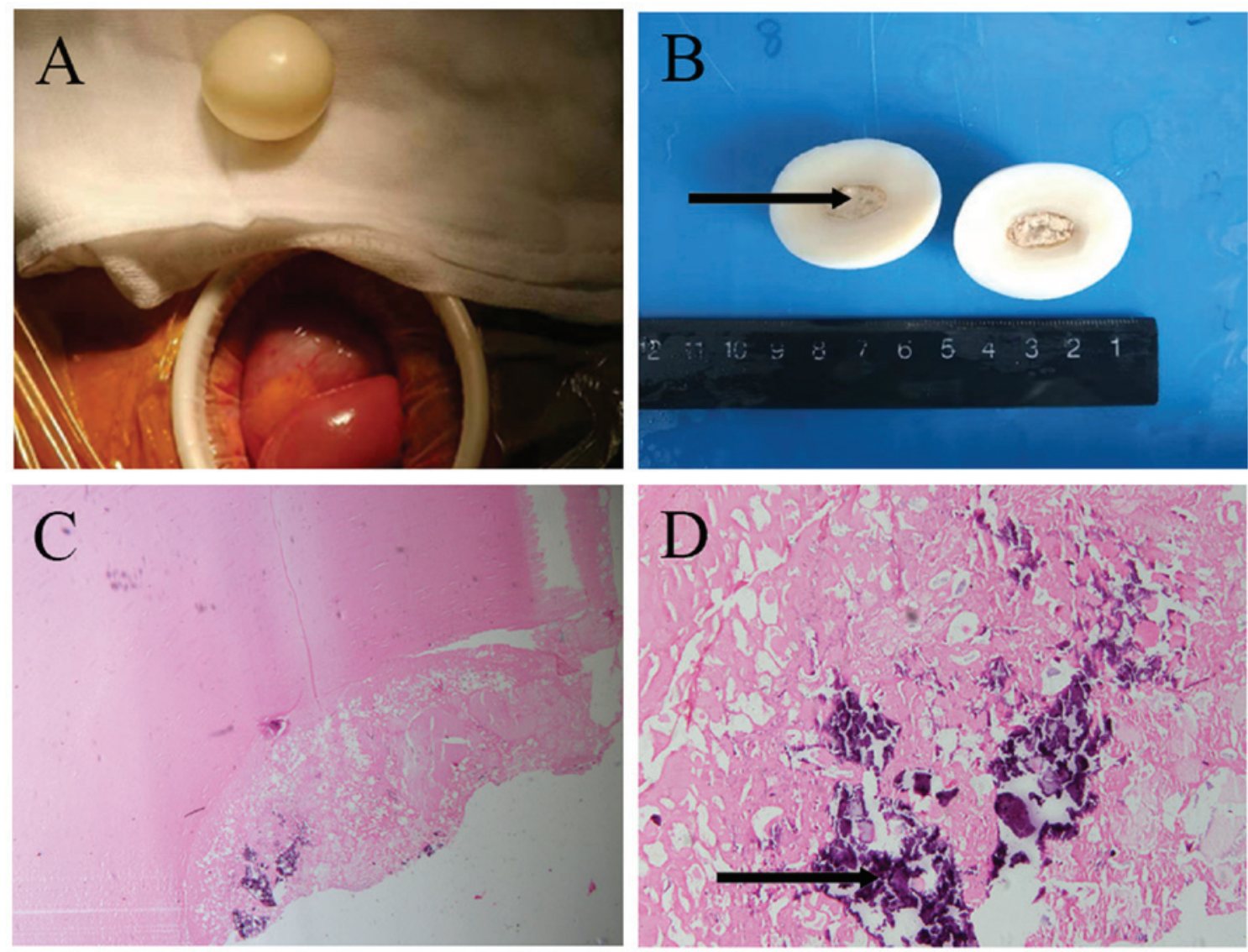

Figure 2. Pathological findings of the tumor. (A) Intra-operative image revealing the extremely regular shape of this tumor. (B) Diameter of the tumor was $\sim 4 \mathrm{~cm}$ and the cross-section of the lesion showed substantial calcification, indicated by the black arrow. (C) H\&E staining revealing the dense collagenous fibrous tissue. Magnification, x20. (D) H\&E staining revealing the calcification in the center area of the tumor. Magnification, x100. H\&E, hematoxylin and eosin.

seen. There were also scattered inflammatory infiltrates, plasma cells, lymphocytes and mast cells. Spindle cells were dispersed among thick collagen bundles (14). Immunohistochemically, CFP cells were diffusely positive for vimentin and factor XIIIa, while focally positive for
SMA, CD34 and desmin. Antibodies against CD117 and S100 were negative. Compared with other countries, tumors of China patients showed higher staining positivity for SMA and CD34. However, only two patients were tested for factor XIIIa. Our statistics suggest that vimentin and 
Table II. Tumor characteristics.

\begin{tabular}{lccc}
\hline Characteristic & China $(\%)$ & Other countries $(\%)$ & Total $(\%)$ \\
\hline Tumor size, cm & & & 0.5 \\
Min & 1 & 0.5 & 10 \\
Max & 7.8 & 10 & $3.7 \pm 2.3$ \\
Average (mean \pm SD) & $2.8 \pm 1.7$ & $4.0 \pm 2.5$ & $12(18.7)$ \\
Location & & & $19(29.7)$ \\
Thorax & $2(12.5)$ & $10(20.8)$ & $13(20.3)$ \\
Stomach & $7(41.2)$ & $12(25.0)$ & $8(12.5)$ \\
Intestine & $1(6.3)$ & $12(25.0)$ & $6(9.4)$ \\
Abdominal cavity & $2(12.5)$ & $6(12.5)$ & $6(9.4)$ \\
Pelvic or perineum & $3(18.8)$ & $3(7.3)$ & \\
Other & $1(6.3)$ & $5(10.4)$ & $30 / 30(100.0)$ \\
Immunoreactive & & & $15 / 15(100.0)$ \\
Vimentin & $14 / 14(100.0)$ & $16 / 16(100.0)$ & $11 / 27(40.7)$ \\
Factor XIIIa & $2 / 2(100.0)$ & $13 / 13(100.0)$ & $20 / 42(47.6)$ \\
SMA & $8 / 10(80.0)$ & $3 / 17(17.6)$ & $1 / 29(3.4)$ \\
CD34 & $11 / 14(78.6)$ & $9 / 28(32.1)$ & $6 / 29(20.7)$ \\
CD117 & $0 / 10(0.0)$ & $1 / 19(5.3)$ & $1 / 32(3.1)$ \\
Desmin & $6 / 10(60.0)$ & $0 / 19(0.0)$ & $0 / 28(0.0)$ \\
S-100 protein & $1 / 4(25.0)$ & &
\end{tabular}

Table III. Tumor immunohistochemistry.

\begin{tabular}{|c|c|c|c|c|c|}
\hline Immunostain & CFP & GIST & IMT & SFT & Leiomyoma \\
\hline Factor XIIIa & + & $\mathrm{N}$ & + & + & - \\
\hline Vimentin & + & + & + & + & + \\
\hline SMA & \pm & \pm & + & \pm & + \\
\hline CD34 & \pm & \pm & \pm & + & - \\
\hline CD117 & - & + & - & - & - \\
\hline Desmin & \pm & - & - & - & + \\
\hline S-100 protein & - & - & - & - & - \\
\hline
\end{tabular}

CFP, calcifying fibrous pseudotumor; GIST, gastrointestinal stromal tumor; IMT, inflammatory myofibroblastic tumor; SFT, solitary fibrous tumors; N, not applicable; +, positive; -, negative; \pm positive or negative.

factor XIIIa are valuable immunohistochemical mark of CFP. Improving rates of examination for vimentin and factor XIIIa might be valuable for the diagnosis of CFP.

The differential diagnosis of CFP is difficult. We listed the widely used immunostains for CFP and other potential differential diagnoses (Table III). For patients presenting with abdominal discomfort, gastrointestinal stromal tumors (GISTs) and inflammatory myofibroblastic tumors (IMTs) should be considered first. GIST originating from the interstitial cells of Cajal are the most common single type of sarcoma in China. They are mostly seen in stomach (50-60\%) and small intestine (30-35\%). More than $80 \%$ of the patients were over 50 years old. The clinical symptoms were mild (15). Their characteristics include lack of hyalinized collagen, calcification lesions and staining positive for CD117, CD34 and anoctamin 1 were the main differences from CFP. IMT was possibly related to genetic predisposition and infection (16). The tumors were abundant in fibrous tissue, consisting of variable quantities of plasma cells, lymphocytes, eosinophils, foamy histiocytes, and mast cells. IMT could distinguished from CFP by expression of anaplastic lymphoma kinase and rearrangement of chromosome 2p23 (6). Moreover, CFP from pleura had to be differentiated from other pleural lesions such as solitary fibrous tumors (SFTs). SFTs were often positive for CD34 and had no dystrophic calcifications (17). Leiomyoma and calcifying aponeurotic fibroma should also be considered in the differential diagnoses of tumors in the musculoskeletal system. Leiomyoma has calcifications and ossifications, and its cells are positive for desmin, SMA, caldesmon, and factor XIIIa. Calcifying aponeurotic fibromas are less circumscribed 
and usually occur in the hands and feet. Metaplastic cartilage and multinuclear giant cells are typically (18).

In conclusion, CFPs are rare benign tumors that are difficult to diagnose preoperatively. We found that tumors were more common in patients aged from 18 to 60 years. They were immunoreactive for vimentin and factor XIIIa. In China, the most common location of CFP was the stomach. Perhaps this is related to the eating habits of the Chinese. Nevertheless, the number of reported cases is low. Clinical features and pathogenesis of this disease need to be characterized in further studies.

\section{Acknowledgements}

Not applicable.

\section{Funding}

The present study was supported by The National Natural Science Foundation of China (grant no.30972877), The Science and Technology Commission of Shanghai Municipality (grant no. 16ZR1400800) and The Cohort Study of Colorectal Cancer (grant no. 2017YFC0908200).

\section{Availability of data and materials}

The datasets used and/or analyzed during the present study are available from the corresponding author on reasonable request.

\section{Authors' contributions}

HYM was responsible for the analysis of data and the writing of the manuscript. MTF contributed to acquisition of the materials and the design of the study. YGH was responsible for the conception of the manuscript, design of the study and checking of the primary data. All authors read and approved the final manuscript.

\section{Ethics approval and consent to participate}

The present study was approved by The Institutional Review Board of Ethics Committee of Changhai Hospital (Shanghai, China). The patient gave consent to be included in the present study.

\section{Patient consent for publication}

The patient gave written consent for image and data publication.

\section{Competing interests}

The authors declare that they have no competing interests.

\section{References}

1. Rosenthal NS and Abdul-Karim FW: Childhood fibrous tumor with psammoma bodies. Clinicopathologic features in two cases. Arch Pathol Lab Med 112: 798-800, 1988

2. Saglam EA, Usubütün A, Kart C, Ayhan A and Küçükali T: Reactive nodular fibrous pseudotumor involving the pelvic and abdominal cavity: A case report and review of literature. Virchows Arch 447: 879-882, 2005.

3. Hill KA, Gonzalez-Crussi F, Omeroglu A and Chou PM: Calcifying fibrous pseudotumor involving the neck of a five-week-old infant. Presence of factor XIIIa in the lesional cells. Pathol Res Pract 196: 527-531, 2000.

4. Xu L, Zhu H, Yang Y and Bao J: Imaging features of calcifying fibrous tumor. Chin J Med Imaging 24: 298-302, 2016.

5. Prucker J, Salaheddin-Nassr Y and Leidl S: Calcifying fibrous tumor of the terminal ileum mesentery: Case report. Medicine (Baltimore) 97: e13351, 2018

6. Wu T, Zhu P, Duan X, Yang X and Lu D: Calcifying fibrous pseudotumor of the adrenal gland: A rare case report. Mol Clin Oncol 5: 252-254, 2016.

7. Özkan S, Demırağ F, Yekeler E and Karaoğlanoğlu N: Calcifying fibrous pseudotumor of lungs. Turk J Med Sci 44: 901-903, 2014.

8. Kang W, Cui Z, Li X, Sun P and Jin X: Calcifying Fibrous Tumor of the Tunica Vaginalis Testis: A report of 2 cases. Urology 100: e9-e13, 2017.

9. Larson BK, Balzer B, Goldwasser J and Dhall D. Calcifying fibrous tumor: An unrecognized IgG4-Related disease. APMIS 123: 72-76, 2015

10. Shimizu S, Funakoshi Y, Yoon HE, Okuma T, Utsumi T, Ito N, Sakaguchi M, Taniguchi K, Eimoto T and Matsumura A: Small calcifying fibrous pseudotumor of the heart confined to the epicardium. Cardiovasc Pathol 24: 191-193, 2015.

11. Mehrad M, LaFramboise WA, Lyons MA, Trejo Bittar HE and Yousem SA: Whole-exome sequencing identifies unique mutations and copy number losses in calcifying fibrous tumor of the pleura: Report of 3 cases and review of the literature. Hum Pathol 78: 36-43, 2018.

12. Wesecki M, Radziuk D, Niemiec S, Waniczek D and Lorenc Z: Calcifying fibrous tumor of the small bowel mesentery in a 27-year old male patient-case report. Pol Przegl Chir 86: 436-439, 2014.

13. Fan SF, Yang H, Li Z and Teng GJ: Gastric calcifying fibrous pseudotumour associated with an ulcer: Report of one case with a literature review. Br J Radiol 83: e188-e191, 2010.

14. Li BJ, Yang XD, Chen WX, Shi YH, Nie ZH and Wu J: Calcifying fibrous tumor of stomach: A case report. Medicine (Baltimore) 96: e8882, 2017.

15. Joensuu $\mathrm{H}$, Hohenberger $\mathrm{P}$ and Corless CL: Gastrointestinal stromal tumour. Lancet 382: 973-983, 2013.

16. Liu HK, Lin YC, Yeh ML, Chen YS, Su YT and Tsai CC: Inflammatory myofibroblastic tumors of the pancreas in children: A case report and literature review. Medicine (Baltimore) 96: e5870, 2017.

17. Ağaçkıran Y, Fındık G, Aydoğdu K, Günay E, Günay S and Kaya S: An extremely rare case of multiple calcifying tumor of the pleura. Tuberk Toraks 60: 385-388, 2012.

18. Hoffmann H, Beaver ME and Maillard AA. Calcifying fibrous pseudotumor of the neck. Arch Pathol Lab Med 124: 435-437, 2000. 